\title{
Magnetic Resonance Imaging in Pediatric Bone Tumors
}

\author{
Flavio Duarte Silva $\cdot$ Lucypaula Pinheiro • \\ Carlos Cristofano $\cdot$ Jose Luiz de Oliveira Schiavon • \\ Henrique Manoel Lederman
}

Published online: 29 October 2014

(c) Springer Science+Business Media New York 2014

\begin{abstract}
The primary assessment of pediatric bone lesions is performed using radiographic imaging and is categorized according to criteria of aggressiveness. Magnetic resonance imaging plays a complementary diagnostic role in select cases, for example, when there are characteristics of fatty content, cysts with and without fluid-fluid levels, cartilaginous matrix, and low signal intensity on T2 sequences. In bone lesions presenting an aggressive pattern, MRI is the chosen method for locoregional staging and non-invasive evaluation of response to neoadjuvant treatment.
\end{abstract}

Keywords MRI $\cdot$ Pediatrics $\cdot$ Bone tumors

This article is part of the Topical Collection on MRI for Pediatric Oncology.

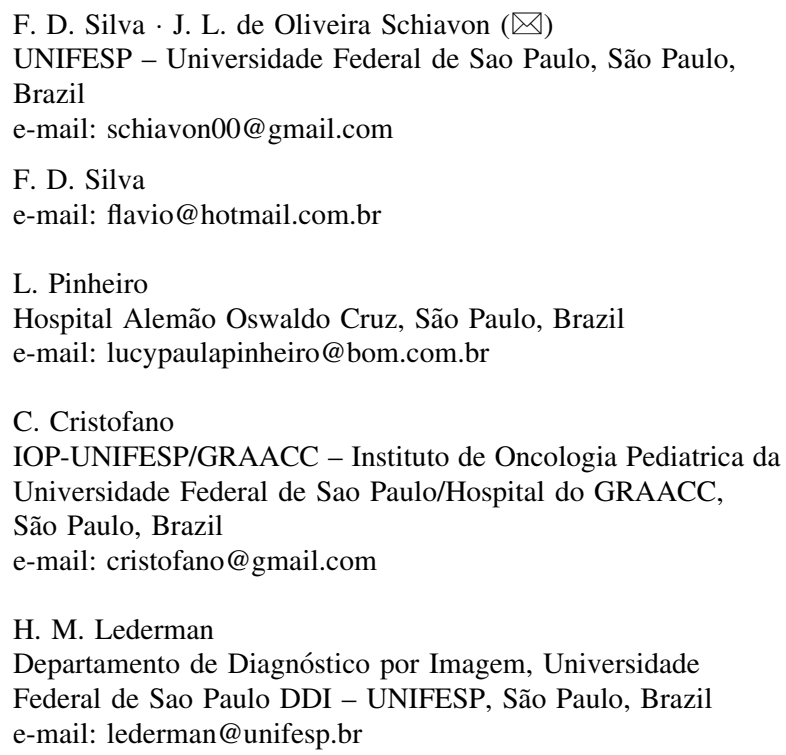

\section{Introduction}

The term bone tumor is a broad category which includes neoplasms, tumor-like conditions, reactive focal abnormalities, and metabolic diseases [1•].

The neoplastic processes can be categorized as benign and malignant, and the latter may be subcategorized as primary and secondary. It is estimated that primary benign tumors are ten times more common than primary malignant tumors. Despite encompassing more than twenty different histological varieties, the majority of primary malignant tumors are osteosarcomas (OS-52 \%) and Ewing's sarcomas (ES-34 \%) [2, 3].

The secondary bone involvement by metastasis or primary bone tumors, such as chondrosarcoma, is common among adults, whereas it is an exception among children. Inversely, lesions such as eosinophilic granuloma, simple bone cysts (SBCs), and aneurysmal bone cysts (ABCs) are typical in children.

Numerous imaging techniques are available for the assessment of bone tumors; however, radiography is still the primary screening technique and the least expensive method for the detection and histological characterization of most tumors or tumor-like bone conditions $[1 \bullet, 4,5]$. The radiographic characteristics of bone tumors provide essential information for the definition of aggressiveness, mainly by the way of analyzing the margins of the lesion, according to Lodwick's classification, the presence and type of periosteal reaction, and cortical modifications $[1 \bullet$, 5]. Therefore, even in cases of unspecific lesions identified primarily with magnetic resonance imaging (MRI), conventional radiography should be used.

Following up an accurate evaluation of the radiographs in conjunction with knowledge of the patient's age, sex, and clinical presentation cannot be overemphasized because it 

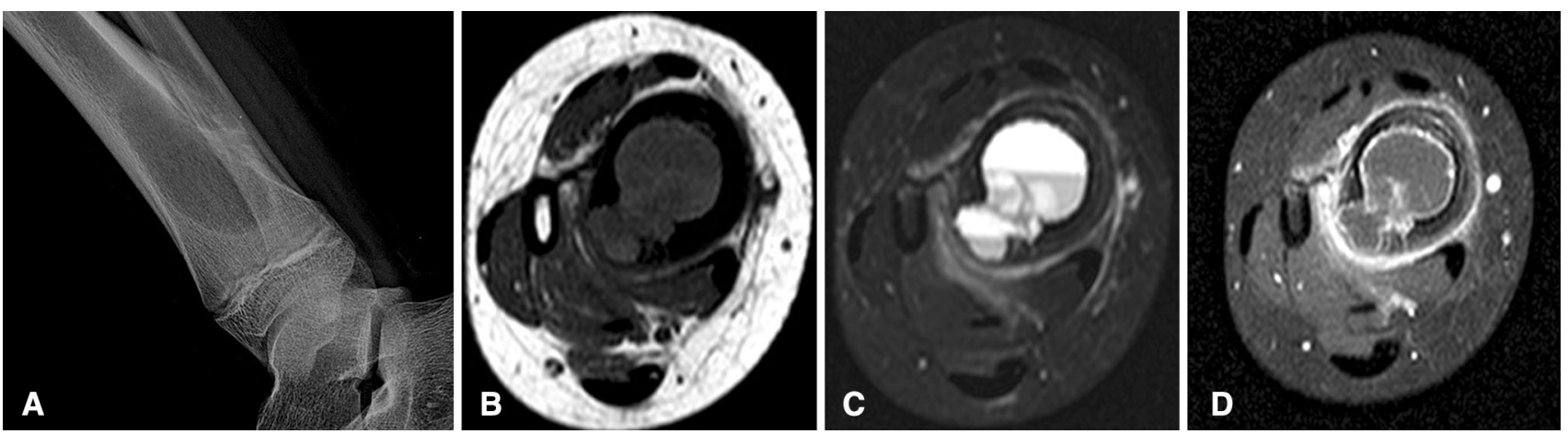

Fig. 1 X-ray with geographic margin without periosteal reaction, suggesting lesion with low aggressiveness. However, image shows tapering and apparent cortical rupture in the posterior aspect (a). MRI was performed for better analysis of the posterior aspect of the lesion,

determines the next step in the evaluation of the abnormality. Patient management follows one of four paths: [1•] no future observation or treatment required (for example, metaphyseal fibrous defect); [2] observation with periodic plain film examination (e.g., enchondroma); [3] biopsy and excision (e.g., benign intracompartmental lesions); or [4] further imaging for staging before biopsy and surgery (e.g., aggressive benign lesions, indeterminate, and malignant tumors) [4]. Other imaging studies may be useful in further characterization of lesions in groups 2 and 3.

The limitations of radiographic assessment relate to the low contrast of non-mineralized tissue which results in low sensitivity in detecting extension to the soft tissues. Despite the trabecular bone constituting a mineralized tissue, intramedullary extension is also limited in the radiographic study. This is even more evident in aggressive processes, in which the tumor growth wave exceeds the velocity of the trabecular lysis, as well as the fact that radiographic study can only identify trabecular lysis when there is a 30-40\% bone mineral destruction. These two factors can cause the true intramedullary extension of the lesion to be underestimated.

By providing high contrast of tissue and proving superior to $\mathrm{CT}$ imaging in the intra and extraosseous evaluation of lesions [6••, 7], MRI is the chosen modality to glean more indepth information regarding the extent of the process, especially in cases of suspected malignant tumor. MRI is regarded as the most effective method for local pre-operative staging $[7-9,10 \bullet, 11,12]$. Besides this, the analysis of signal intensity (SI) using a variety of MRI may add propaedeutic data, narrowing down the diagnostic possibilities (Fig. 1).

\section{Technical Aspects of MRI}

There are two main groups of MRI indications in the initial approach of focal bone lesions. The first is lesions with in which characteristics of cyst were demonstrated, with a high signal on T2 (c), low signal on T1 (b) and peripheral enhancement after GdCM(D). This was a complicated simple bone cyst with fracture. X-ray profile (a), axial T1 (b), axial T2 (c), and axial T1 post-contrast (d)

characteristics of low aggressiveness but with undetermined etiology in which more detailed content is sought, such as SI on T1 and T2, solid or cystic nature, the presence of fluid-fluid level or fat, findings which can consolidate the idea of conservative activity, or in some cases guide local resection surgery or curettage.

The second group contains lesions which present aggressive characteristics and will undergo biopsy (ideally performed after MRI), and whose objective is locoregional staging.

MRI evaluation should begin with localization sequences, including longitudinal images of the whole bone containing lesion and its proximal and distal articulations. In the group of lesions with radiographic characteristics showing aggressiveness, the longitudinal sequence should maintain a wide field of view (FOV) and inclusion of the articulations, avoiding an inaccurate staging assessment such as that caused by a skip lesion, and hindering the surgical management and limb salvage.

The assessment should analyze the following sequences in the axial and longitudinal planes: T1-weighted spin echo (T1W SE), T2-weighted fast spin echo (T2W FSE) and its variation with fat saturation (T2W FSE FS) or STIR (short tau inversion recovery), T1-weighted spin echo with fat saturation (T1W SE FS), and this same sequence after intravenous gadolinium contrast media injection (T1W SE FS Gd-CM) with focus on maintaining repetition time (TR) and echo time (TE) on T1W images before and after Gd-CM.

T1W SE sequence produces excellent contrast between cortical bone, bone marrow, and surrounding soft tissues, as well as relatively high spatial resolution. Most bone tumors are readily evident as well-defined regions of low signal against a background of surrounding fatty marrow. Although T1W images also can be obtained with FSE or gradient-echo (GE) pulse sequences, virtually all the articles that have described the signal characteristics of various bone tumors have reported on T1W SE. 
T2W FSE and STIR sequences can be considered to produce a "pathology scan," which shows high signal within most tumors due to their increased water content. Frequency-selective FS becomes essential when using these FSE sequences, as they often show both fat and tumor with similar, relatively high SI.

Contrast-enhanced MRI is important in characterization of bone tumors, as well as in assessment of response to therapy and detection of recurrent tumor. A Gd-CM accumulates in well-vascularized tissues and it manifests as increased SI on T1W images. Because fat also has high signal on T1W images, it is essential to apply frequencyselective FS with both pre- and post-Gd enhanced images. In some cases, it can be helpful to subtract the pre-contrast images from the post-contrast images to emphasize the regions that enhanced [13]

The advanced MRI techniques using diffusion and perfusion sequences are indicated in staging lesions and may also be used in some specific cases of non-aggressive lesions.

\section{Advanced MRI Techniques}

For some patients, conventional MR imaging is unable to provide a specific histological diagnosis or determine the true extent of tumor necrosis, which is used to assess response to treatment and the prognosis [14]. These patients may benefit from advanced MR imaging techniques, such as diffusion-weighted imaging (DWI), dynamic contrast-enhanced (DCE) perfusion imaging, and in phase/opposed-phase MR imaging.

MRI diffusion-weighted images (DWI) demonstrate the Brownian stochastic motion of water molecules on a microscopic level within tissue and DWI signal is derived from motion of water molecules in the extracellular, intracellular, and transcellular space, as well as in the intravascular space (micro-circulation-perfusion fraction) [15]. DWI is, therefore, the reflection of the microscopic tissue structure, and the motion of water molecules is more restricted in tissues with high cellularity, intact cell membranes, and reduced extracellular space.

DWI is able to measure diffusivity by the application of diffusion sensitizing gradients to T2-weighted images, currently most commonly via single shot echo planar imaging (SS-EPI) sequences.

DWI is normally carried out using at least two $b$ values (e.g.,: $b=0 \mathrm{~s} / \mathrm{mm}^{2}$ and another value of $b$, between 0 and $1,000 \mathrm{~s} / \mathrm{mm}^{2}$ ) to allow a significant interpretation. The qualitative analysis of DWI is in the visual analysis of SI of different $b$ values. For example, the cystic or necrotic section of a tumoral lesion will produce a lower SI in images of $a$ high $b$ value because the diffusion of the water molecules is less restricted, in contrast, the more solid cellular tumors will continue to show a relatively high SI.

The quantitative analysis is represented by the apparent diffusion coefficient (ADC: units: $\mu \mathrm{m}^{2} / \mathrm{s}$ or $10^{-3} \mathrm{~mm}^{2} / \mathrm{s}$ ). Areas of restricted diffusion (i.e., because of high cellularity) have high SI on DWI but lower SI on ADC maps.

DCE imaging provides physiologic information that cannot be determined from conventional MR imaging, including information regarding tissue vascularization and perfusion, capillary permeability, and the volume of the interstitial space.

DCE MR imaging is performed with fast (usually volumetric) GE sequences that are prescribed over a section or volume of interest and repeated several times after intravenous contrast agent administration, to exploit the contrast-enhancement properties of a tumor. An intravenous injection of $0.1 \mathrm{mmol} / \mathrm{Kg}$ of gadolinium-based contrast agent is performed using an infusion pump at a rate of $2-5 \mathrm{~mL} / \mathrm{s}$, followed by $20 \mathrm{ml}$ of saline solution, and imaging takes place with a temporal resolution of 5-30 s for approximately 3-7 min. With this technique, quantitative information on lesion enhancement can be displayed graphically in a time-intensity curve, by measuring signal intensities in one or more circular or freely determined regions-of-interest (ROI) following a bolus injection of $\mathrm{Gd}$ CM. Pixel-by-pixel post-processing techniques create parametric images that display the contrast-enhancement ( =subtraction images) or physiologic information, like the initial ( =first-pass images) and delayed rate of contrast agent accumulation ( =wash-out images).

Chemical shift MRI may be useful in distinguishing benign and malignant marrow processes, mainly by identifying whether the processes contain fat [16॰]. A $20 \%$ decrease in SI on the opposed-phase images relative to that on the in-phase images is a reliable quantitative metric for distinguishing benign from malignant bone marrow in the spine [17-19], but caution should be used, provided the utility of chemical shift imaging is likely more important in distinguishing a true marrow- replacing tumor from an infiltrative process such as bone marrow edema, hematopoietic marrow, or other infiltrative lesions rather than for strictly distinguishing benign and malignant bone tumors.

\section{Role of MRI in Bone Tumors}

\section{Characterization}

There is considerable overlapping in the radiographic characteristics of different histological groups of bone lesions, and in this context, some MRI findings may provide valuable information in order to reduce the diagnostic differentials. 

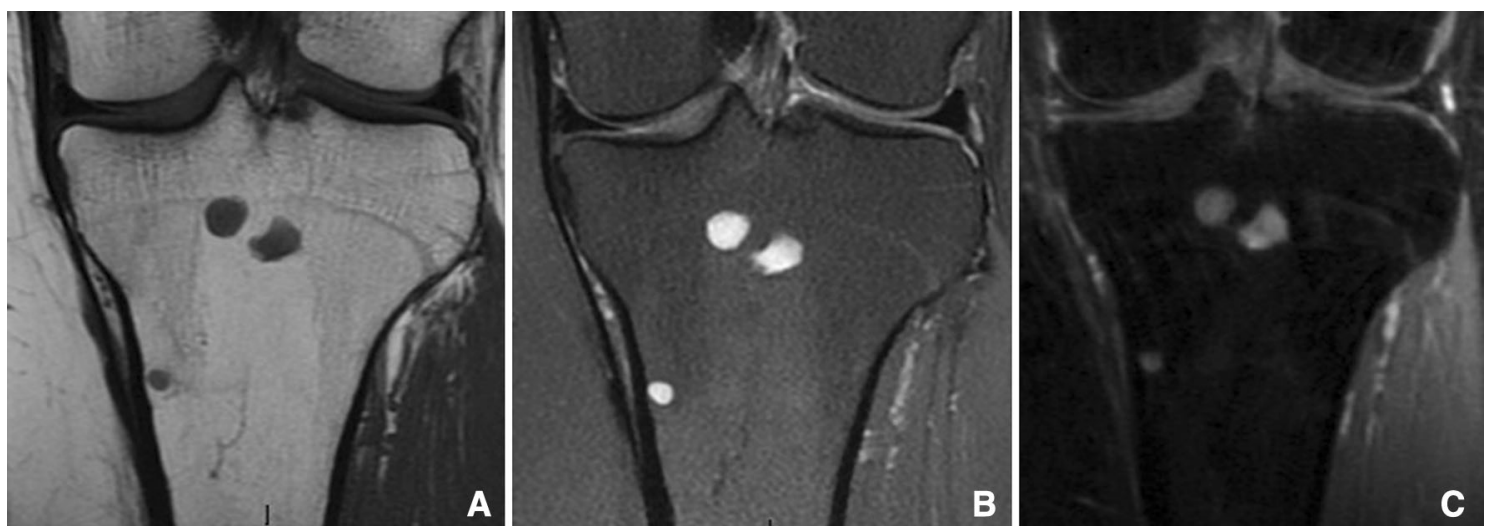

Fig. 2 Intraosseous lipoma Coronal T1 (a), coronal T2 (b) and coronal $\mathrm{T} 1$ post-contrast (c). Lipoma in the metadiaphyseal region with hyperintense signal on $\mathrm{T} 1$ without fat saturation (a) and with hypointense signal on $\mathrm{T} 2$ with fat saturation (b) and without

\section{Fat SI Within Tumors}

The presence of intratumoral fat is confirmed by hyperintense signal on $\mathrm{T} 1 \mathrm{~W}$ images, which is suppressed on the T1W FS without contrast, reinforcing the need for the latter sequence in protocol of osseous tumors. In the absence of T1W FS images, in order to consider the possibility of fat content, the lesion must show the same high SI in T1W and loss of SI in the T2W or STIR sequences; however, this same signal activity may occur due to the subacute hematic component or melanin.

The majority of lesions containing fat are benign, including hemangioma and intraosseous lipoma. Lipomas are the principal fatty bone lesions, either entirely or partially composed of fatty content, and may concomitantly present cystic components (Fig. 2), fatty necrotic areas, dystrophic ossifications, and fluid-fluid level [20-23]. Lipomas are most commonly found in the proximal region of the femur (transtrochanteric and subtrochanteric region) and the calcaneus. Parosteal lipomas are very rare and appear as just a cortical fatty masses accompanied by osseous excrescences and cortical thickening at the base [24]. Osseous liposarcomas are rare and may also contain fat, however, they usually present signs of aggressiveness $[25,26]$.

Hemangiomas, which also contain internal fat, are permeated by thick bony trabeculae [27].

Certain primary bone lesions may also undergo fatty change with time such as ABCs and SBCs, particularly in the calcaneum [22, 28]. Malignant lesions such as metastasis and lymphomas may undergo fatty involution following radiotherapy [29] and in rare cases, chemotherapy.

Medullary bone infarcts are non-neoplastic lesions that typically demonstrate peripheral serpiginous sclerosis with a central area of fat SI [30••] enhancement by contrast (c), cystic degenerative foci are also seen in the superior medial aspect, characterized by high signal on T2 and low signal on $\mathrm{T} 1$ and peripheral enhancement by contrast

\section{Cartilaginous Lesions}

Cartilaginous neoplastic lesions have characteristic morphological and SI features in MRI. These tumors are typically present with lobulated margins which are isointense to muscle on $\mathrm{T} 1 \mathrm{~W}$ images and markedly hyperintense on T2W or STIR images, they commonly contain internal and peripheral hypointense vascular septa, which enhance with gadolinium. Another characteristic that may be seen is punctate areas of signal void, due to matrix mineralization. Such features typify low-grade tumors as chondroma (Fig. 3), atypical chondroma, and low-grade (grade 1) chondrosarcoma.

Despite the rarity of chondrosarcomas in pediatric patients, some signs should be observed in order to differentiate between high and low degree lesions. In relation to the intramedullary chondral lesions, Murphy et al. conclude that the most reliable features suggestive of chondrosarcoma are endosteal scalloping greater than $2 / 3$ the thickness of the cortex, cortical destruction, extension to the epiphysis, and soft tissue extension. A concentric growth pattern rather than lobular growth, size greater than 5-6 cm, and areas of heterogeneous low T2W may also suggest malignancy [31]. Furthermore, whereas chondromas have more frequently inhomogeneous peripheral or central enhancement, chondrosarcomas have low signal septa, which separate the high signal lobules and enhance markedly in a ring and arc fashion [32]. Chondrosarcomas of all grades can show early enhancement (within $10 \mathrm{~s}$ ), with some demonstrating exponential enhancement on dynamic-enhanced gradient- echo (GRE) MRI. Chondromas demonstrate no $(>2 \mathrm{~min})$ or late $(10 \mathrm{~s}-2 \mathrm{~min})$ enhancement with a gradual increase in enhancement. Absence of enhancement is useful in excluding malignancy, whereas early and exponential enhancement is suggestive of chondrosarcoma [33]. 

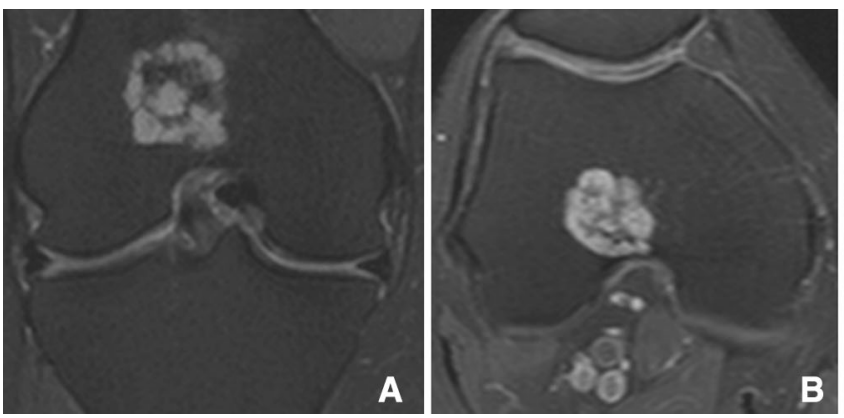

Fig. 3 Endochroma in the distal metaphyseal region of the fêmur with lobulated contours, heterogeneous with areas of hyperintense signal on sagital T2 (a) and axial T2 (b) permeated by low signal foci

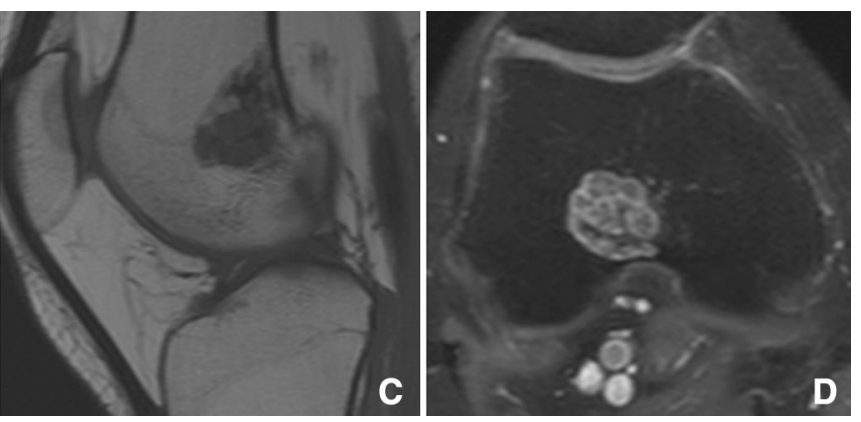

on T1 (c) and T2 (mineralized matrix), with no posterior cortical tapering (b, c), presenting predominantly peripherical and septal lobular enhancement (d)
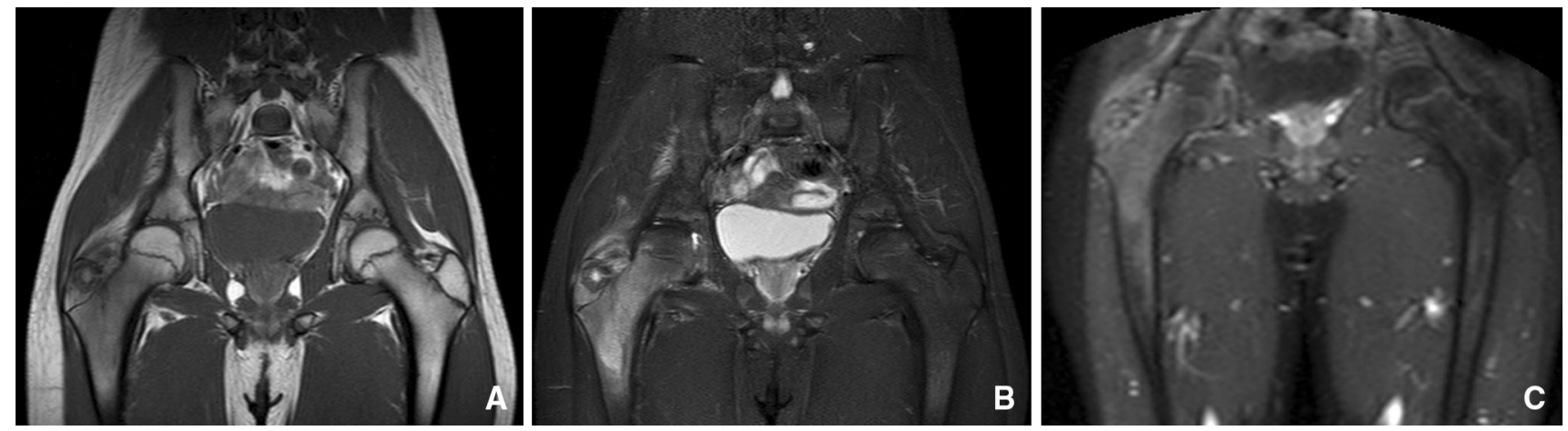

Fig. 4 Lesion compromising right greater trochanter apophysis with heterogeneous signal on T1 and T2 (a, b), associated with adjacent bone marrow edema, also compromising the femoral neck with hyperintense signal on T2 (b) and enhancement post-Gd (c). Chondroblastoma

Contrary to all other cartilaginous bone lesions, chondroblastoma more typically demonstrates low to intermediate heterogeneous SI on T1W and T2W images, lobular internal architecture and fine lobular margins on MRI [34] (Fig. 4). Chondroblastoma are epiphyseal lesions, and such as osteoid osteoma, frequently are surrounded by bone marrow edema.

Osteochondromas have well-recognized imaging features. An exostotic bone lesion that is continuous with the underlying marrow cavity is identified. A cartilage cap is often demonstrated, which is low to intermediate SI on T1W and hyperintense on T2W/STIR sequences (Fig. 5) [30••]. Malignant transformation occurs in the cartilage cap, and cap thickness of greater than $20 \mathrm{~mm}$ in children, as depicted optimally on axial T2W MRI, is the best predictor of malignant change to chondrosarcoma [35].

\section{Cystic Lesions}

Bone cysts are characterized upon the identification of only a thin rim of peripheral enhancement. The SBCs often have a low SI on T1 and a high SI on T2 homogenous [13], and the ABCs commonly present fluid-fluid levels [36]. In both, T1 shortening may reflect haemorrhagic or proteinaceous content resulting in signal which is higher than water and in SBCs, fluid-fluid levels (FFLs) are usually seen following fracture [37]. Other changes following fracture of cystic lesions include apparently solid areas and a variety of enhancement patterns, including focal nodular, heterogeneous central, a thick peripheral rim, and subcortical with adjacent soft tissue enhancement (Fig. 1) [13, 37].

\section{Fluid-Fluid Levels}

FFLs may occur whenever substances of differing densities are contained within a cystic or compartmentalized structure (Fig. 1) [38]. Usually, they are related to prior hemorrhage or tumor necrosis. The prevalence of FFLs in bone lesions is $2.7-11.2 \%[36,39]$ and is most commonly reported in $\mathrm{ABCs}$, but they have been identified in many different types of bone tumors, both benign and malignant, leading to the conclusion that they are a non-specific finding. Secondary ABC formation within a primary bone lesion (e.g., giant cell tumor, osteoblastoma, chondroblastoma, and fibrous dysplasia), which occurs with a frequency of up to $35 \%$ in several large series, may be the cause of the large number of bone tumors that may present with FFLs [39]. 

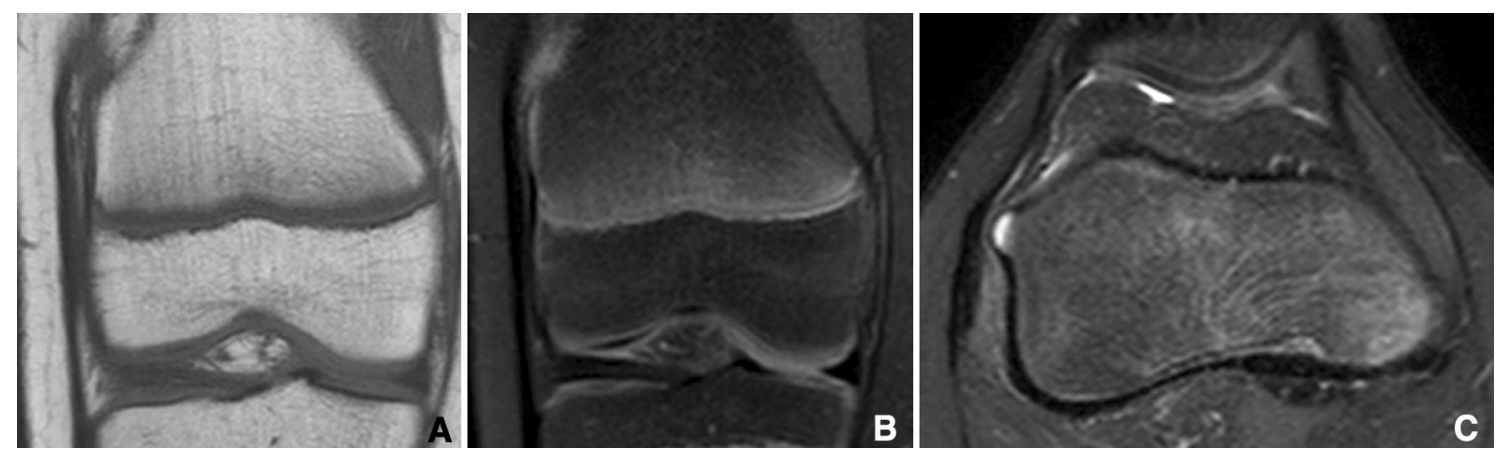

Fig. 5 11-year-old male with distal lateral thigh pain and radiography (unavailable) with small lateral exostosis of the distal fêmur. Due to the extent of pain, MRI was requested to evaluate the complication of the osteochondroma. MRI T1 W characterizes small lateral exostosis (a). T2W images $(\mathbf{b}, \mathbf{c})$ identify the cartilage cap with no signs of transformation, with thickness of $3.0 \mathrm{~mm}$, and show signs of friction between the osteochondroma and the iliotibial tract with edema in the interjacent myoadipose plane $(\mathbf{b}, \mathbf{c})$
The proportion of the lesion filled with FFLs may be related to the likelihood of malignancy. As the proportion of the lesion occupied by FFLs increases, so does the frequency of benign diagnoses. Conversely, if a lesion contains less than $1 / 3$ FFLs, it is more likely to be malignant, most commonly conventional central OS [39].

\section{Fibrous Matrix and Hemosiderin Deposition}

Tissues may demonstrate low SI on both T1W and T2W sequences, including osteoblastic or fibrous matrix or due the paramagnetic effects of hemosiderin. Fibrous tumors, as non-ossifying fibroma (NOF) (Fig. 6) and desmoplastic fibroma of bone most commonly have hypointensity on T2W images, and SI are dependent on the amounts of hypercellular fibrous tissue, collagen, and bone trabeculae $[40,41]$.

Giant cell tumor frequently demonstrates hypointensity on $\mathrm{T} 2 \mathrm{~W}$ images related to hemosiderin deposition from recurrent hemorrhage. This may demonstrate a nodular, zonal, whorled, or diffuse pattern [42].

Long-standing lesions that have sustained repeated trauma may also present hemosiderin deposition and low SI on $\mathrm{T} 2 \mathrm{~W}$, such as SBC, fibrous dysplasia, and haemangioma.

\section{Locoregional Staging}

Accurate pre-operative local staging techniques allow planning of limb-saving surgery in patients with primary bone sarcoma, and MRI has surpassed CT and angiography in this task $[7,8,43,44 \cdot \bullet]$.

MRI is mainly important in determining the longitudinal intraosseous extent of the tumor, and its relationship with the physis, joint, muscle compartments, and neurovascular bundles.
The objective of analysis of the intraosseous extension is to determine the proximal and distal limits of the lesions and correlate them with surgical reference points, aiding in the surgical planning, and is best performed in the longitudinal plane on T1 (Fig. 7) [9, 10].

Surgeon may prefer to leave the epiphysis intact with a segmental (intercalary) resection when possible, sparing the physis and growth potential. Furthermore, leaving the epiphysis tends to preserve the natural joint and a better functional outcome [45]. The parallel analysis of T1 and STIR images indicates epiphyseal extension of osteosarcoma as a low SI on T1 and a high SI on STIR or by disturbance of the normal architecture of the physis or epiphysis. Peritumoral effects (possible edema, hematopoietic marrow, or angiogenesis) usually show an intermediate signal on T1 or STIR imaging, have indistinct margins, and do not disturb the physeal or epiphyseal architecture. Although observations of T1W and STIR sequences related to epiphyseal involvement were equally accurate, STIR was more sensitive overall and T1 was more specific [45].

Longitudinal T1W SE images, by using a large FOV, were found to have a higher sensitivity than conventional radiography and bone scintigraphy for the detection of skip lesions in patients with bone sarcoma [8]. A skip metastasis (Fig. 7) is suspected whenever one or more nodular foci of hypointense signal are identified on $\mathrm{T} 1 \mathrm{~W}$, anatomically separate from the primary lesion and occurring either within the same bone, or across the adjacent joint, then it is categorized as a trans-articular skip [46]

Intra-articular extension of bone sarcomas is one criterion that determines whether a patient is a candidate for an intraor extraarticular resection. Extraarticular en bloc resection is required in cases of tumor extension into the joint space, which results in inferior post-operative function 

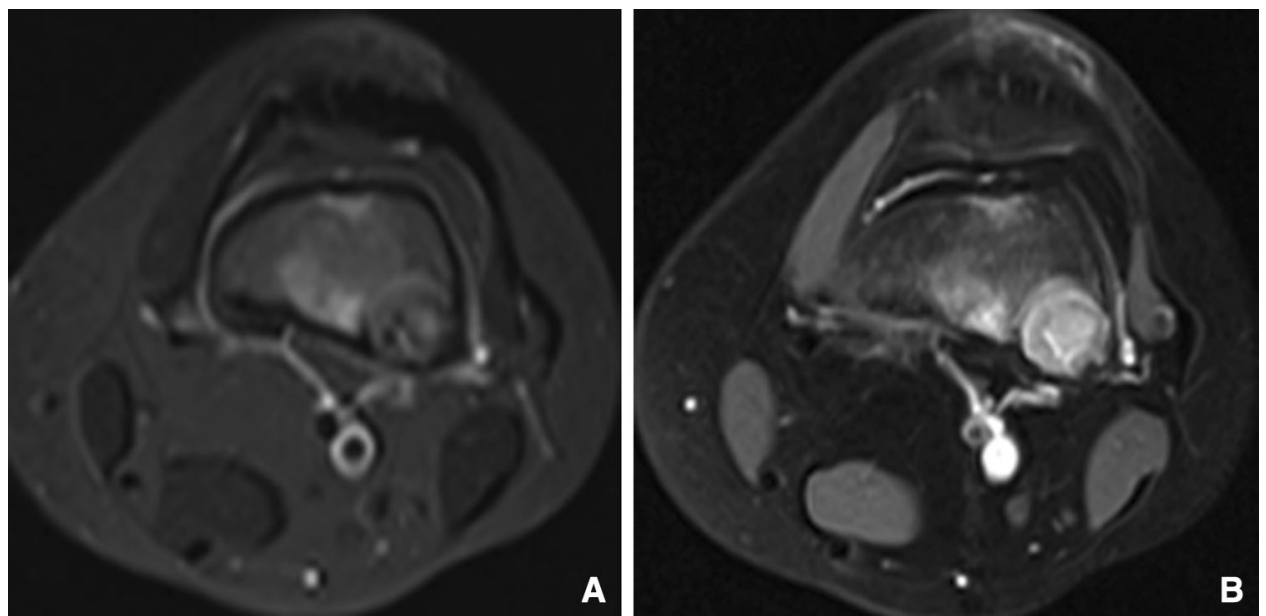

Fig. 6 Excentric post-erolateral metadiaphyseal lesion of the distal fêmur with low signal matrix on STIR (a) favoring the diagnosis of fibrous matrix (non-ossifying fibroma). There was diffusive impregnation of the lesion by Gd-CM (b). Non-ossifying fibroma
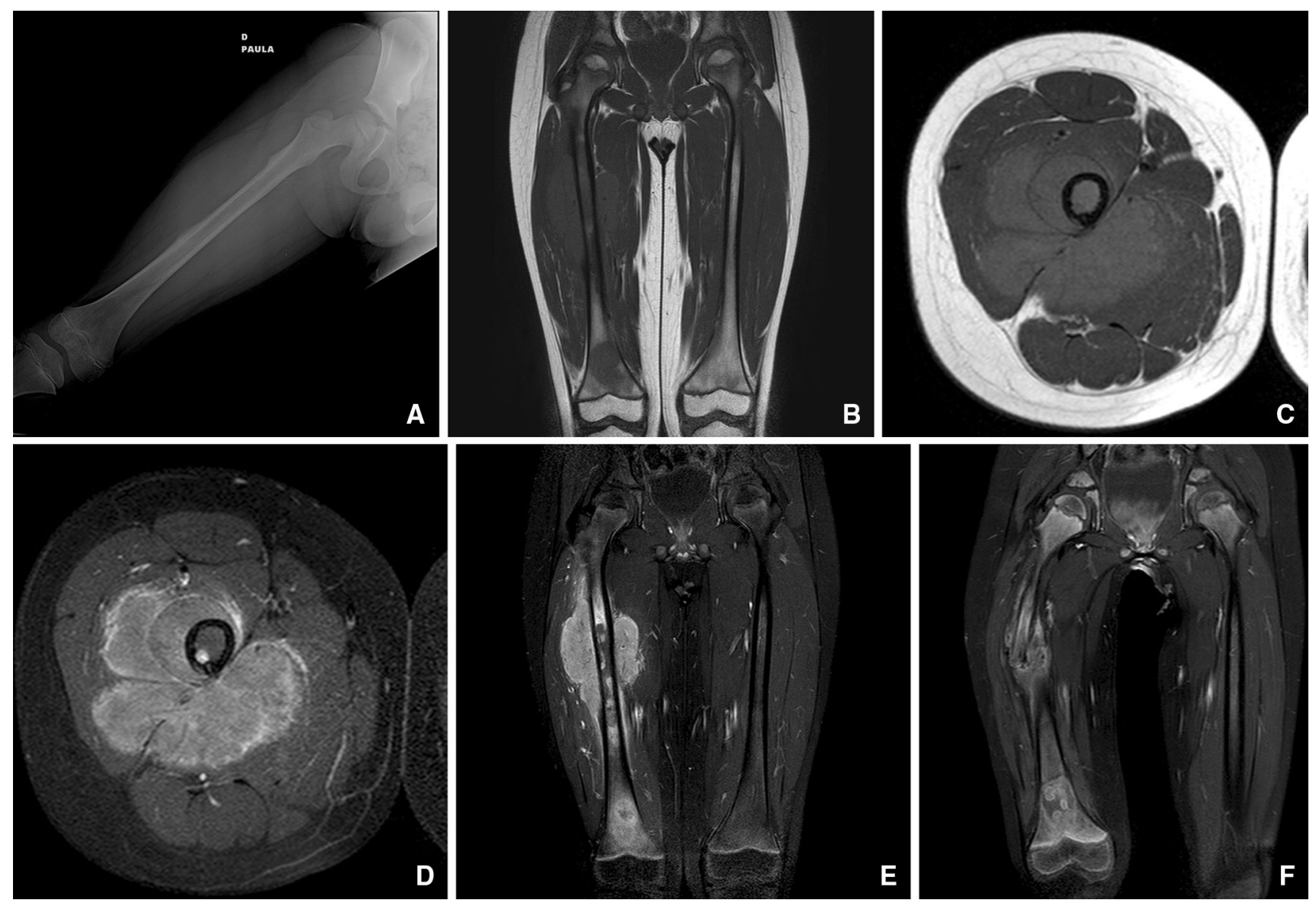

Fig. 7 6-year-old male experiencing pain and thigh edema for 2 months and fever for a week. X-ray displays interrupted periosteal reaction (a). MRI was carried out for staging with wide FOV images showing an aggressive lesion with bone marrow involvement and extension to adjacent soft tissues on $\mathrm{T} 1(\mathbf{b}, \mathbf{c})$ and $\mathrm{T} 1$ pos-Gd $(\mathbf{d}$,

e) with no cleavage plane with femoral vessels (d) and the presence of distal metadiaphyseal skip metastasis of the fêmur (b, e, f). The patient underwent chemotherapy, to which he showed a good response, with a volumetric reduction greater than $75 \%$ of the tumor (e, f). Ewing's sarcoma 

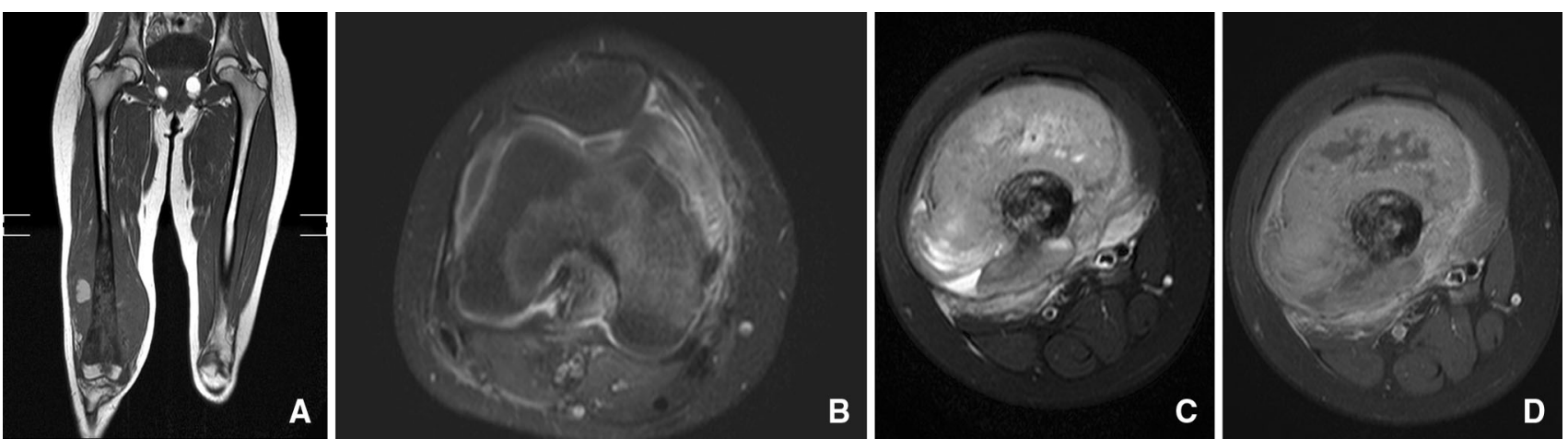

Fig. 8 Lesion in the metadiaphyseal region of the femur, with extension to soft tissues and to the epiphyseal region on coronal T1. a There is also intra-articular extension of the lesion with involvement of the cortical bone and capsule, as well as tumoral enhancement in the adjacent extraosseous and intra-articular soft tissues on axial T1

Considering the presence of tumoral enhancement exceeding the capsular and synovial plane or cortical and articular cartilage as articular invasion (Fig. 8), or in the case of the knee, intracapsular and extrasynovial enhancement of the anterior cruciate ligament, MRI showed sensitivity of $100 \%$ but limited specificity (69\%). The absence of joint effusion is significant because of its high negative predictive value ( $92 \%$ ); however, its presence should not be interpreted as articular tumoral extension, being detected in up to $69 \%$ of patients without articular involvement [47].

Extraosseous extension encompasses the assessment of muscle compartments, fascia, adipose tissue, and relation of the tumor to large neurovascular bundles. For this purpose, sequences which display greater contrast between tumoral mass and all other surrounding tissues, especially the neurovascular bundle are useful. T1W SE post-Gd enhanced images, when compared with T2W SE images, rated better for differentiation between tumor and muscle and between tumor and fat, and was also considered superior in demonstrating the relationship to the neurovascular bundle. Neurovascular bundle may be classified as "not involved" when a clear interface is observed between the tumor and the bundle structures, "equivocal" when a thin layer of normal tissue is interposed, and "involved" when they are contiguous (no free tissue plane can be visualized on one or more images) (Fig. 7). Using these criteria, Van Trommel et al. were always correct when MRI indicated the vessels were not involved. With equivocal involvement, vessels were involved in approximately $50 \%$ of cases, and when vascular involvement was diagnosed on MRI, they were correct in three out of four cases [48].

\section{Monitoring Neoadjuvant Chemotherapy}

The gold standard for the assessment of response to preoperative chemotherapy is the histopathological analysis of
post-Gd. b In this case, the femoral vessels pass adjacent to the lesion with no tissue preservation between the two $(\mathbf{b}, \mathbf{d})$. The assessment of the surgical specimen showed no invasion of the neurovascular bundle. Osteosarcoma

the resected tumor that separates good and poor respondents among patients with osteosarcoma and Ewing's sarcoma [44••, 49]. Several histological classifications have been proposed, always based on the percentage of tumor necrosis and viable-appearing tumor. It is an independent major prognostic parameter for relapse-free and overall survival in patients with osteogenic sarcoma [50] and Ewing's sarcoma [51].

The imaging prediction of response to chemotherapy may allow modification of neoadjuvant treatment protocols in poor respondents, select patients for the performance and timing of limb-salvage surgery, plan the radiation therapy, and select the post-operative chemotherapy regimens [44••].

Prediction of response to preoperative chemotherapy with MRI is primarily based on changes in gross morphology, such as tumor size and margins, on changes in SI and enhancement patterns after injection of contrast agents, or on changes in DWI $[52,53]$.

Evaluation of response by way of MRI follows the fundamental principle of attempting to differentiate viable areas of tumor from all other intratumoral areas, which include areas of liquefaction necrosis, hemorrhagic areas, granulation tissue, and fibrosis.

Static contrast-enhanced images allow the differentiation of vascularized tumoral areas (e.g., viable tumor cells, granulated, or fibrous tissue) and non-vascularized areas (e.g., liquefaction necrosis). However, these images do not distinguish the viable tumor from immature vascularized granulation tissue, fibrous tissue, neovascularity in necrotic areas, and reactive hyperemia [44••].

MRI protocols including static contrast-enhanced have been unable to provide an accurate quantitative measurement of tumor response $[54,55]$. In these cases, the evaluation of response by MRI focuses on the volumetric change in tumor (OS and ES) and signal on T2W images. An increase in OS e ES volume is highly suggestive of poor respondents, on the other hand decrease in OS volume 
does not allow separation between good and poor respondents [56, 57]. In ES, a reduction of less than $25 \%$ indicates poor respondents, between 25 and $75 \%$ there is an overlap between groups, and greater than $75 \%$ correlates to good histopathological respondents (Fig. 7).

OS poor respondents could also be identified if no decrease in the amount of edema is seen or if there is increase T2W SI of the extraosseous tumor component $[57,58]$.

DCE MRI studies try to characterize tumor components that do not enhance as much as cellular viable-appearing tumor areas, therefore, differentiating between good and poor respondents. It is the method of choice to monitor preoperative chemotherapy. Poor and good responders to chemotherapy can be detected on first-pass or subtraction images, or by evaluation of changes in slope values using the ROI method. It provides important information for presurgical monitoring of response to neoadjuvant chemotherapy and may also be useful in selected cases for assessment of local recurrence of tumor [44••, 59]

DWI is a promising method in the evaluation of necrosis to monitor pre-operative chemotherapy, since the diffusion signal is directly related to the tumor cellularity, necrotic areas within the tumoral tissue induce a local diffusion increase. This phenomenon has been demonstrated in clinical practice [60,61]. Ideally, quantitative evaluation (ADC) should be carried out in the initial exam, half way through the course of chemotherapy [62] and at the end of neoadjuvant chemotherapy [63, 64].

\section{Conclusion}

Radiography is an imaging tool at the forefront of identification, determination of degree of aggressiveness, and ranking of diagnostic differentials in bone lesions. In non-aggressive and radiographically unspecific lesions, MRI may provide greater specificity in the diagnosis through signal patterns captured in its wide array of modalities and by enhanced pattern, in which case being more informative than dynamic sequences. In aggressive lesions, MRI is the optimum method for locoregional staging and has shown promising results in determining the degree of tumoral necrosis both during and after chemotherapy treatment, by utilizing advanced sequences.

\section{Compliance with Ethics Guidelines}

Conflict of Interest Dr. Flavio Duarte Silva, Dr. Lucypaula Pinheiro, Dr. Carlos Cristofano, and Dr. Jose Luiz de Oliveira Schiavon each declare no potential conflicts of interest. Dr. Henrique Manoel Lederman is a section editor for Current Radiology Reports.

Human and Animal Rights and Informed Consent This article does not contain any studies with human or animal subjects performed by any of the authors.

\section{References}

Recently published papers of particular interest have been highlighted as:

- Of importance

•• Of major importance

1. - Miller TT. Bone tumors and tumorlike conditions: analysis with conventional radiography. Radiology. 2008;246(3):662-74. This article reviews radiographic aspects in the analysis of bone tumors.

2. Stiller CA, Bielack SS, Jundt G, Steliarova-Foucher E. Bone tumours in European children and adolescents, 1978-1997. Report from the automated childhood cancer information system project. Eur J Cancer. 2006;42(13):2124-35.

3. Eyre R, Feltbower RG, Mubwandarikwa E, Eden TOB, McNally RJQ. Epidemiology of bone tumours in children and young adults. Pediatr Blood Cancer. 2009;53(6):941-52.

4. Sundaram M, McLeod RA. MR imaging of tumor and tumorlike lesions of bone and soft tissue. AJR Am J Roentgenol. 1990;155(4):817-24.

5. Costelloe CM, Madewell JE. Radiography in the initial diagnosis of primary bone tumors. AJR Am J Roentgenol. 2013;200(1):3-7.

6. - Wetzel LH, Levine E, Murphey MD. A comparison of MR imaging and CT in the evaluation of musculoskeletal masses. Radiographics. 1987;7(5):851-74. The authors compare MRI and CT evaluation of bone and soft tissue masses, and they found MRI to be equal or superior to $C T$.

7. Bloem JL, Bluemm RG, Taminiau AH, van Oosterom AT, Stolk J, Doornbos J. Magnetic resonance imaging of primary malignant bone tumors. Radiographics. 1987;7(3):425-45.

8. Bloem JL, Taminiau AH, Eulderink F, Hermans J, Pauwels EK. Radiologic staging of primary bone sarcoma: MR imaging, scintigraphy, angiography, and CT correlated with pathologic examination. Radiology. 1988;169(3):805-10.

9. Onikul E, Fletcher BD, Parham DM, Chen G. Accuracy of MR imaging for estimating intraosseous extent of osteosarcoma. AJR Am J Roentgenol. 1996;167(5):1211-5.

10. • Gillespy T, Manfrini M, Ruggieri P, Spanier SS, Pettersson H, Springfield DS. Staging of intraosseous extent of osteosarcoma: correlation of preoperative CT and MR imaging with pathologic macroslides. Radiology. 1988;167(3):765-7. The authors evaluated accuracy of preoperative computed tomography (CT) and magnetic resonance (MR) imaging in the measurement of intraosseous tumor extent and compared with macroslides of surgical specimens. The authors conclude that MR imaging is extremely accurate in the assessment of intraosseous extent of osteosarcoma.

11. van Trommel MF, Kroon HM, Bloem JL, Hogendoorn PC, Taminiau AH. MR imaging based strategies in limb salvage surgery for osteosarcoma of the distal femur. Skelet Radiol. 1997;26(11):636-41.

12. Mirowitz SA, Apicella P, Reinus WR, Hammerman AM. MR imaging of bone marrow lesions: relative conspicuousness on T1weighted, fat-suppressed T2-weighted, and STIR images. AJR Am J Roentgenol. 1994;162(1):215-21.

13. Davies AM, Sundaram M, James SJ, editors. Imaging of bone tumors and tumor-like lesions: techniques and applications (medical radiology/diagnostic imaging). 2009th ed. Berlin: Springer; 2009.

14. Costa FM, Canella C, Gasparetto E. Advanced magnetic resonance imaging techniques in the evaluation of musculoskeletal tumors. Radiol Clin North Am. 2011;49(6):1325-58, vii-viii.

15. Koh D-M, Takahara T, Imai Y, Collins DJ. Practical aspects of assessing tumors using clinical diffusion-weighted imaging in the body. Magn Reson Med Sci. 2007;6(4):211-24. 
16. - Fayad LM, Jacobs MA, Wang X, Carrino JA, Bluemke DA. Musculoskeletal tumors: how to use anatomic, functional, and metabolic MR techniques. Radiology. 2012;265(2):340-56. The authors review MRI techniques in the evaluation of bone and soft tissue tumors.

17. Zajick DC Jr, Morrison WB, Schweitzer ME, Parellada JA, Carrino JA. Benign and malignant processes: normal values and differentiation with chemical shift MR imaging in vertebral marrow. Radiology. 2005;237(2):590-6.

18. Zampa V, Cosottini M, Michelassi M, Ortori S, Bruschini L, Bartolozzi C. Value of opposed-phase gradient-echo technique in distinguishing between benign and malignant vertebral lesions. Eur Radiol. 2001;12(7):1811-8.

19. Disler DG, McCauley TR, Ratner LM, Kesack CD, Cooper JA. In-phase and out-of-phase MR imaging of bone marrow: prediction of neoplasia based on the detection of coexistent fat and water. AJR Am J Roentgenol. 1997;169(5):1439-47.

20. Propeck T, Bullard MA, Lin J, Doi K, Martel W. Radiologicpathologic correlation of intraosseous lipomas. AJR Am J Roentgenol. 2000;175(3):673-8.

21. Milgram JW. Intraosseous lipomas: radiologic and pathologic manifestations. Radiology. 1988;167(1):155-60.

22. Campbell RSD, Grainger AJ, Mangham DC, Beggs I, Teh J, Davies AM. Intraosseous lipoma: report of 35 new cases and a review of the literature. Skeletal Radiol. 2003;32(4):209-22.

23. Murphey MD, Carroll JF, Flemming DJ, Pope TL, Gannon FH, Kransdorf MJ. From the archives of the AFIP. Radiographics. 2004;24(5):1433-66.

24. Murphey MD, Johnson DL, Bhatia PS, Neff JR, Rosenthal HG, Walker CW. Parosteal lipoma: MR imaging characteristics. AJR Am J Roentgenol. 1994;162(1):105-10.

25. Torigoe T, Matsumoto T, Terakado A, Takase M, Yamasaki S, Kurosawa $\mathrm{H}$. Primary pleomorphic liposarcoma of bone: MRI findings and review of the literature. Skeletal Radiol. 2005;35(7):536-8.

26. Murphey MD, Arcara LK, Fanburg-Smith J. Imaging of musculoskeletal liposarcoma with radiologic-pathologic correlation. Radiographics. 2005;25(5):1371-95.

27. Wenger DE, Wold LE. Benign vascular lesions of bone: radiologic and pathologic features. Skeletal Radiol. 2000;29(2):63-74.

28. Wada R, Lambert RGW. Deposition of intraosseous fat in a degenerating simple bone cyst. Skeletal Radiol. 2004;34(7):415-8.

29. Vanel D, Bittoun J, Tardivon A. MRI of bone metastases. Eur Radiol. 1998;8(8):1345-51.

30. •• Alyas F, James SL, Davies AM, Saifuddin A. The role of MR imaging in the diagnostic characterisation of appendicular bone tumours and tumour-like conditions. Eur Radiol. 2007;17(10): 2675-86. This article reviews the value of MRI in the further characterisation of appendicular bone tumours and tumour-like lesions, with particular reference to peri-lesional oedema, fluid-fluid levels, flow voids, fat signal, cartilage signal and dedifferentiation.

31. Murphey MD, Flemming DJ, Boyea SR, Bojescul JA, Sweet DE, Temple HT. Enchondroma versus chondrosarcoma in the appendicular skeleton: differentiating features. Radiographics. 1998;18(5):1213-37; quiz 1244-5.

32. De Beuckeleer LH, De Schepper AM, Ramon F, Somville J. Magnetic resonance imaging of cartilaginous tumors: a retrospective study of 79 patients. Eur J Radiol. 1995;21(1):34-40.

33. Geirnaerdt MJ, Hogendoorn PC, Bloem JL, Taminiau AH, van der Woude HJ. Cartilaginous tumors: fast contrast-enhanced MR imaging. Radiology. 2000;214(2):539-46.

34. Weatherall PT, Maale GE, Mendelsohn DB, Sherry CS, Erdman WE, Pascoe HR. Chondroblastoma: classic and confusing appearance at MR imaging. Radiology. 1994;190(2):467-74.

35. Woertler K, Lindner N, Gosheger G, Brinkschmidt C, Heindel W. Osteochondroma: MR imaging of tumor-related complications. Eur Radiol. 2000;10(5):832-40.
36. Van Dyck P, Vanhoenacker FM, Vogel J, Venstermans C, Kroon HM, Gielen J, et al. Prevalence, extension and characteristics of fluid-fluid levels in bone and soft tissue tumors. Eur Radiol. 2006;16(12):2644-51.

37. Margau R, Babyn P, Cole W, Smith C, Lee F. MR imaging of simple bone cysts in children: not so simple. Pediatr Radiol. 2000;30(8):551-7.

38. Tsai JC, Dalinka MK, Fallon MD, Zlatkin MB, Kressel HY. Fluid-fluid level: a nonspecific finding in tumors of bone and soft tissue. Radiology. 1990;175(3):779-82.

39. O'Donnell P, Saifuddin A. The prevalence and diagnostic significance of fluid-fluid levels in focal lesions of bone. Skelet Radiol. 2004;33(6):330-6.

40. Jee WH, Choe BY, Kang HS, Suh KJ, Suh JS, Ryu KN, et al. Nonossifying fibroma: characteristics at MR imaging with pathologic correlation. Radiology. 1998;209(1):197-202.

41. Frick MA, Sundaram M, Unni KK, Inwards CY, Fabbri N, Trentani $\mathrm{F}$, et al. Imaging findings in desmoplastic fibroma of bone: distinctive $\mathrm{T} 2$ characteristics. AJR Am J Roentgenol. 2005;184(6):1762-7.

42. Aoki J, Tanikawa H, Ishii K, Seo GS, Karakida O, Sone S, et al. MR findings indicative of hemosiderin in giant-cell tumor of bone: frequency, cause, and diagnostic significance. AJR Am J Roentgenol. 1996;166(1):145-8.

43. Zimmer WD, Berquist TH, McLeod RA, Sim FH, Pritchard DJ, Shives TC, et al. Bone tumors: magnetic resonance imaging versus computed tomography. Radiology. 1985;155(3):709-18.

44. • van der Woude HJ, Bloem JL, Hogendoorn PC. Preoperative evaluation and monitoring chemotherapy in patients with highgrade osteogenic and Ewing's sarcoma: review of current imaging modalities. Skelet Radiol. 1998;27(2):57-71. In this article, conventional and new imaging modalities are discussed with regard to the monitoring of the effect of neoadjuvant chemotherapy.

45. Hoffer FA, Nikanorov AY, Reddick WE, Bodner SM, Xiong X, Jones-Wallace D, et al. Accuracy of MR imaging for detecting epiphyseal extension of osteosarcoma. Pediatr Radiol. 2000;30(5): 289-98.

46. Saifuddin A. The accuracy of imaging in the local staging of appendicular osteosarcoma. Skelet Radiol. 2002;31(4):191-201.

47. Schima W, Amann G, Stiglbauer R, Windhager R, Kramer J, Nicolakis M, et al. Preoperative staging of osteosarcoma: efficacy of MR imaging in detecting joint involvement. AJR Am J Roentgenol. 1994;163(5):1171-5.

48. van Trommel MF, Kroon HM, Bloem JL, Hogendoorn PC, Taminiau AH. MR imaging based strategies in limb salvage surgery for osteosarcoma of the distal femur. Skelet Radiol. 1997;26(11):636-41.

49. Huvos AG. Bone tumors: diagnosis, treatment, and prognosis. 2nd ed. Philadelphia: Saunders; 1990.

50. Petrilli AS, Gentil FC, Epelman S, Lopes LF, Bianchi A, Lopes $A$, et al. Increased survival, limb preservation, and prognostic factors for osteosarcoma. Cancer. 1991;68(4):733-7.

51. Picci P, Bacci G, Campanacci M, Gasparini M, Pilotti S, Cerasoli $S$, et al. Histologic evaluation of necrosis in osteosarcoma induced by chemotherapy. Regional mapping of viable and nonviable tumor. Cancer. 1985;56(7):1515-21.

52. Fletcher BD. Response of osteosarcoma and Ewing sarcoma to chemotherapy: imaging evaluation. AJR Am J Roentgenol. 1991;157(4):825-33.

53. Erlemann R, Sciuk J, Bosse A, Ritter J, Kusnierz-Glaz CR, Peters $\mathrm{PE}$, et al. Response of osteosarcoma and Ewing sarcoma to preoperative chemotherapy: assessment with dynamic and static MR imaging and skeletal scintigraphy. Radiology. 1990;175(3):791-6.

54. Lawrence JA, Babyn PS, Chan HS, Thorner PS, Pron GE, Krajbich IJ. Extremity osteosarcoma in childhood: prognostic value of radiologic imaging. Radiology. 1993;189(1):43-7. 
55. van der Woude HJ, Bloem JL, Holscher HC, Nooy MA, Taminiau AH, Hermans $J$, et al. Monitoring the effect of chemotherapy in Ewing's sarcoma of bone with MR imaging. Skelet Radiol. 1994;23(7):493-500.

56. Holscher HC, Bloem JL, Nooy MA, Taminiau AH, Eulderink F, Hermans J. The value of MR imaging in monitoring the effect of chemotherapy on bone sarcomas. AJR Am J Roentgenol. 1990;154(4):763-9.

57. Holscher HC, Bloem JL, Vanel D, Hermans J, Nooy MA, Taminiau $\mathrm{AH}$, et al. Osteosarcoma: chemotherapy-induced changes at MR imaging. Radiology. 1992;182(3):839-44.

58. Holscher HC, Bloem JL, van der Woude HJ, Hermans J, Nooy MA, Taminiau AH, et al. Can MRI predict the histopathological response in patients with osteosarcoma after the first cycle of chemotherapy? Clin Radiol. 1995;50(6):384-90.

59. Verstraete KL, Lang P. Bone and soft tissue tumors: the role of contrast agents for MR imaging. Eur J Radiol. 2000;34(3):229-46.

60. Nonomura Y, Yasumoto M, Yoshimura R, Haraguchi K, Ito S, Akashi T, et al. Relationship between bone marrow cellularity and apparent diffusion coefficient. J Magn Reson Imaging. 2001;13(5):757-60.

61. Humphries PD, Sebire NJ, Siegel MJ, Olsen ØE. Tumors in pediatric patients at diffusion-weighted MR imaging: apparent diffusion coefficient and tumor cellularity. Radiology. 2007;245(3):848-54.

62. Baunin C, Schmidt G, Baumstarck K, Bouvier C, Gentet JC, Aschero A, et al. Value of diffusion-weighted images in differentiating mid-course responders to chemotherapy for osteosarcoma compared to the histological response: preliminary results. Skelet Radiol. 2012;41(9):1141-9.

63. Oka K, Yakushiji T, Sato H, Hirai T, Yamashita Y, Mizuta H. The value of diffusion-weighted imaging for monitoring the chemotherapeutic response of osteosarcoma: a comparison between average apparent diffusion coefficient and minimum apparent diffusion coefficient. Skelet Radiol. 2010;39(2):141-6.

64. Hayashida Y, Yakushiji T, Awai K, Katahira K, Nakayama Y, Shimomura $\mathrm{O}$, et al. Monitoring therapeutic responses of primary bone tumors by diffusion-weighted image: Initial results. Eur Radiol. 2006;16(12):2637-43. 